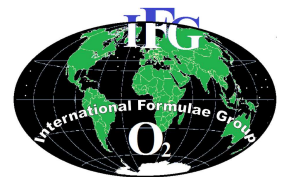

Available online at http://ajol.info/index.php/ijbcs

Int. J. Biol. Chem. Sci. 8(3): 891-902, June 2014

International Journal

of Biological and

Chemical Sciences

ISSN 1997-342X (Online), ISSN 1991-8631 (Print)

http://indexmedicus.afro.who.int

\title{
Etude des nématodes phytoparasites du sol et des racines sous quatre niveaux de fertilisation minérale en culture de niébé
}

\author{
Mamoudou TRAORE ${ }^{1 *}$, François LOMPO ${ }^{1}$, Bouma THIO ${ }^{2}$, Badiori OUATTARA ${ }^{1}$, \\ Korodjouma OUATTARA ${ }^{3}$ et Michel SEDOGO ${ }^{1}$ \\ ${ }^{1}$ Institut de l'Environnement et de Recherches Agricoles (INERA), Département Gestion des Ressources \\ Naturelles et des Systèmes de Productions, Laboratoire Sol-Eau-Plantes, 04 BP 8645 Ouagadougou 04, \\ Burkina Faso, Tél. : 0022650340270 ; Fax. : 0022650340271. \\ ${ }^{2}$ Institut de l'Environnement et de Recherches Agricoles (INERA), Département Productions Végétales \\ Laboratoire de Nématologie, Station de Farako bâ, 01 BP 910 Bobo Dioulasso 01, \\ Burkina Faso, Tel. : 22620970960. \\ ${ }^{3}$ Institut de l'Environnement et de Recherches Agricoles (INERA), Département Gestion des Ressources \\ Naturelles et des Systèmes de Productions Station de Recherche de Saria, Laboratoire Sol-Eau-Plantes, \\ BP 10 Koudougou Burkina Faso, Tel. : 22650446509. \\ *Auteur Correspondant ; E-mail : tramadalbela@yahoo.fr
}

\section{RESUME}

Les nématodes du sol et des racines ont été étudiés dans 42 exploitations paysannes utilisant quatre (04) niveaux de fertilisation NPK (14-23-14) en production de niébé (Vigna unguiculata (L.) Walp). Les exploitations paysannes sont situées dans les villages de Saria, Villy et Godin dans le Centre Ouest du Burkina Faso. L'objectif était d'étudier la dynamique des nématodes sous l'influence des pratiques agricoles différentes. Les nématodes du sol et des racines ont été extraits respectivement par les méthodes de l'élutriateur Seinhorst et de l'appareil asperseur de Seinhorst. Dans les différentes exploitations de niébé, 07 espèces de nématodes phytoparasites du sol ont été recensées. Il s'agit des espèces suivantes : Pratylenchus brachyurus, Helicotylenchus multicinctus, Scutellonema cavenessi, Tylenchorhynchus martini, Xiphinema sp., Telotylenchus indicus et Criconemö̈des curvatum. Parmi elles, H. multicinctus et Scutellonema cavenessi représentent la grande majorité des individus. Les nématodes dans les racines du niébé sont représentés principalement par trois espèces : T. multicinctus, $S$. cavenessi et $P$. brachyurus avec des taux d'infestation de 0 à $6 \mathrm{~N} / \mathrm{g}$ de racines. L'utilisation de la fumure minérale n'a pas eu d'influence sur les nématodes dans le sol mais elle a fait baisser la pression des nématodes sur les racines des cultures.

(C) 2014 International Formulae Group. All rights reserved.

Mots clés : Niébé, nématode phytoparasite, racine, fumure minérale.

\section{INTRODUCTION}

Le niébé (Vigna unguiculata (L.) Walp) est une plante bien adaptée aux conditions climatiques de la zone sahélienne. En effet, cette plante à cycle court permet d'assurer une certaine production vivrière, en dépit du déficit pluviométrique que connaît cette zone. Elle constitue une importante denrée de base en Afrique subsaharienne, particulièrement dans les savanes arides de l'Afrique de 
l'Ouest. Ses graines représentent une précieuse source de protéines végétales, de vitamines et de revenus pour l'homme, ainsi que du fourrage pour les animaux. Les feuilles juvéniles et les gousses immatures sont consommées sous forme de légume (Dugje et al., 2009). Au Burkina Faso, le niébé occupe une place importante dans l'alimentation de la population tant en milieu urbain que rural. A partir du début des années 90, la demande en niébé a augmenté progressivement, sous l'effet du changement des habitudes alimentaires et de l'urbanisation mais aussi à cause d'une demande sous régionale en progrès. De 2000 à 2010, la superficie consacrée à la culture du niébé au Burkina Faso est passée de 27098 ha à 121404,98 ha. La production à la même période est passée de 127682 tonnes à 626 119 tonnes (FAO, 2010). Les avantages liés à sa production et la forte demande tant interne qu'à l'exportation placent le niébé dans les filières stratégiques pour la sécurité alimentaire du pays (Kaboré et al., 2010). Cette plante à cycle court (60 jours) permet d'assurer une certaine production vivrière en dépit des contraintes majeures que connait la production agricole du pays. En effet, les aléas climatiques: l'inadaptation des pratiques culturales et la pauvreté des sols constituent les contraintes biophysiques majeures à la production agricole du pays (Sedogo, 1993 ; Bado, 2002 ; Hien, 2004 ; Lompo, 2009).

Ceci justifie la prise en compte de tous les facteurs limitant potentiels de la production agricole, car aucune intervention, à elle seule ne peut conduire à une réponse spectaculaire. Parmi ces facteurs, les nématodes phytoparasites ont été signalés comme de graves entraves aux productions céréalières et maraîchères dans différentes régions du monde (Luc, 1960 ; Mallamaire, 1965 ; Bachelier, 1978 ; Reversat, 1988 ; Bois et al., 2000 ; Bélair, 2005 ; Talwana et al., 2008). En Afrique Subsaharienne, la presque totalité des cultures vivrières et de rente enregistrent des baisses de rendements du fait des nématodes qui les parasitent (Cadet,
1998 ; Weber et al., 1994 ; Seinhorst, 1998 ; Bois et al., 2000). Ces baisses de rendement dues aux nématodes peuvent atteindre 25 à $40 \%$ en l'absence de traitement nématicide (Prot, 1985 ; Weber et al., 1994, Yeates et al., 1994).

L'objectif général de cette étude était d'identifier les nématodes phytoparasites du sol et des racines de la culture du niébé sous des niveaux de fertilisation différentes et de montrer l'influence de ces pratiques agricoles sur les populations de nématodes. De manière spécifique, il s'agissait de déterminer la composition et l'importance des espèces de nématodes sous ces différentes pratiques culturales.

Notre hypothèse est que les pratiques de fertilisation permettent de réduire l'infestation en nématodes du niébé.

\section{MATERIEL ET METHODES \\ Description du milieu d'étude}

Le Plateau Central du Burkina Faso regroupe 17 provinces administratives et couvre environ $26 \%$ du territoire national. Il s'étend sur environ $94.000 \mathrm{~km}^{2}$. La région est à cheval entre la zone sahélienne au Nord et la zone soudano sahélienne au Sud. Il est situé entre $11^{\circ}$ à $14^{\circ}$ de latitude Nord et $0^{\circ}$ à $3^{\circ}$ de longitude Ouest. Il est soumis à un climat de type soudano-sahélien, avec une saison des pluies de quatre à cinq mois et une saison sèche de sept à huit mois. Le couvert végétal est essentiellement constitué de formations secondaires de dégradation (Ouédraogo, 2005).

L'inventaire des nématodes a été réalisé dans 42 champs sous des pratiques culturales différentes situées dans les villages de Saria, Villy et Godin. Les champs de niébé ont été fertilisés en NPK (14-23-14) à quatre doses : 0-20 kg/ha (G I); 20-40 kg/ha (G II); 40-60 kg/ha (G III) ; 60-120 kg/ha (G IV).

\section{Echantillonnage des nématodes}

Les nématodes ont été échantillonnés selon la méthode des cultures en lignes. 
L'échantillon d'une parcelle est constitué par des prélèvements de sols effectués à $20 \mathrm{~cm}$ de profondeur, en plusieurs endroits à l'aide d'une truelle de manière à couvrir toute la parcelle. La superficie des champs de niébé varie de 0,1 à 2 ha. Un échantillon pèse en moyenne $3 \mathrm{~kg}$ de sol et contient des fragments de racines du niébé. Le prélèvement des échantillons a été effectué à maturité du niébé quant la récolte a déjà commencée. Les populations de la plupart des nématodes phytoparasites dans le sol ont tendance à culminer en cette période, une fois que la partie aérienne des cultures s'est fanée ou est morte (Celetti, 2006).

\section{Extraction des nématodes}

L'extraction des nématodes du sol a été faite en utilisant la méthode de l'élutriateur de Seinhorst (Merny et Luc, 1969). Une fraction de sol de $250 \mathrm{~cm}^{3}$ a été prélevée par échantillon et soumise aux trois phases que comporte cette méthode d'extraction: la phase d'élutriation, la phase de tamisage et la phase de filtration active qui sépare les nématodes des impuretés du sol sur la base de leur mobilité. Après extraction, l'identification des nématodes a été effectuée à l'aide de critères biologiques tels que la forme du corps, la forme du stylet et les soies de la tête. Le dénombrement des nématodes a été effectué à l'aide de la plaque de comptage sous la loupe binoculaire. Les effectifs de la population ont été exprimés en nombre de nématodes $/ \mathrm{dm}^{3}$ de sol $\left(\mathrm{N} / \mathrm{dm}^{3}\right)$. L'extraction des nématodes des racines a été faite par la méthode de l'appareil «asperseur» de Seinhorst (Merny et Luc, 1969). L'échantillon des racines a été lavé, découpé en menus morceaux, placé sur un support à grosses mailles et déposé dans un entonnoir relié à un récipient. Les racines ont été maintenues sous un brouillard pendant 14 jours. Leur décomposition a entrâné la libération des nématodes qui ont été retenus dans le récipient. Ils sont soumis à une filtration active avant le comptage. Les effectifs de la population des nématodes sont exprimés en nombre de nématodes / gramme de racines fraîches (N/g racines).

\section{Traitement et analyse des données}

Les données obtenues ont été introduites dans le tableur Excel et soumises à l'analyse de variance (ANOVA), General Linear Model (GLM) en utilisant Minitab version 13.1. Les moyennes ont été comparées à l'aide du test de Fisher, au seuil de probabilité de 5\%.

\section{RESULTATS}

\section{Les nématodes du sol \\ Sur les différentes exploitations de} niébé, 07 espèces de nématodes phytoparasites ont été recensées dans le sol. Il s'agit des espèces suivantes : Pratylenchus brachyurus, Helicotylenchus multicinctus, Scutellonema cavenessi, Tylenchorhynchus martini, Xiphinema sp., Telotylenchus indicus et Criconemoïdes curvatum (Tableaux 1, 2, 4 et 4). Parmi elles, $H$. multicinctus et Scutellonema cavenessi représentent la grande majorité des individus. Après ce premier groupe, deux espèces de nématodes Pratylenchus brachyurus et Tylenchorhynchus martini ont occupé la deuxième place en termes d'importance. Les trois autres espèces, Xiphinema sp., Telotylenchus indicus et Criconemoüdes curvatum ont été très faiblement représentées, avec des densités de population ne dépassant pas $60 \mathrm{~N} / \mathrm{dm}^{3}$.

$\mathrm{Au}$ niveau des treize (13) producteurs du G I (0-20 kg/ha de NPK), nous avons des moyennes d'infestations respectives de 1992 $\mathrm{N} / \mathrm{dm}^{3}$ de sol et $726 \mathrm{~N} / \mathrm{dm}^{3}$ de sol, pour les deux principales espèces $H$. multicinctus et $S$. cavenessi (Tableau 1). Leurs valeurs maximales sont observées chez les producteurs Go20 pour H. multicinctus et Go01 pour $S$. cavenessi, avec, respectivement, $4980 \mathrm{~N} / \mathrm{dm}^{3}$ de sol et $1913 \mathrm{~N} / \mathrm{dm}^{3}$ de sol

L'infestation par les nématodes chez les dix (10) producteurs G II qui ont fertilisé leurs exploitations avec du NPK, aux doses de 
20 à $40 \mathrm{~kg} / \mathrm{ha}$, a montré des valeurs moyennes de $2451 \mathrm{~N} / \mathrm{dm}^{3}$ de sol et $1049 \mathrm{~N} / \mathrm{dm}^{3}$ de sol pour les deux nématodes du sol que sont $H$. multicinctus et $S$ canevessi (Tableau 2). Leurs valeurs maximales ont été de $7180 \mathrm{~N} / \mathrm{dm}^{3}$ de sol pour $H$. multicinctus chez le producteur Sa10 et $2773 \mathrm{~N} / \mathrm{dm}^{3}$ de sol pour $S$. canevessi chez le producteur Vi13. L'infestation par $H$. multicinctus a été supérieure à $1000 \mathrm{~N} / \mathrm{dm}^{3}$ chez 11 des 13 producteurs. Les exceptions ont été observées chez Sa23 et Sa12, avec, respectivement, 427 et $473 \mathrm{~N} / \mathrm{dm}^{3}$ de sol. L'infestation par les cinq autres nématodes a été très faible, avec des taux moyens de moins de $150 \mathrm{~N} / \mathrm{dm}^{3}$ de sol.

$\mathrm{Au}$ niveau des exploitations des trois (03) producteurs du groupe G III (40-60 kg/ha de NPK), on note une population de nématodes du sol dominée toujours par $H$. multicinctus et $S$. cavenessi (Tableau 3). Les valeurs moyennes de ces deux espèces ont été, respectivement, de $2565 \mathrm{~N} / \mathrm{dm}^{3}$ de sol et 605 $\mathrm{N} / \mathrm{dm}^{3}$ de sol. Au sein des exploitations de ce groupe de producteurs, on a noté une plus grande importance de $H$. multicinctus par rapport à $S$. cavenessi. Leurs valeurs maximales ont été, respectivement, de 3933 $\mathrm{N} / \mathrm{dm}^{3}$ de sol (producteur Sa05) et $840 \mathrm{~N} / \mathrm{dm}^{3}$ de sol (producteur Go21). Les 05 autres nématodes ont été relativement très peu représentés et ont eu des taux d'infestation allant de 04 à $98 \mathrm{~N} / \mathrm{dm}^{3}$ de sol.

Les nématodes du sol au sein du G IV (60-120 kg/ha de NPK) ont été également dominés par $H$. multicinctus et $S$. cavenessi tout comme dans les 03 autres cas, avec cependant des valeurs moyennes plus élevées (Tableau 4). On a observé $3649 \mathrm{~N} / \mathrm{dm}^{3}$ de sol en moyenne pour $H$. multicinctus et 1547 $\mathrm{N} / \mathrm{dm}^{3}$ de sol pour $S$. cavenessi. La valeur maximale de $H$. multicinctus s'est située à $7000 \mathrm{~N} / \mathrm{dm}^{3}$ de sol dans l'exploitation de Sa02. Celle de $S$. cavenessi est à $3033 \mathrm{~N} / \mathrm{dm}^{3}$ de sol pour le producteur $\mathrm{Sa} 09$.

Chez l'ensemble des producteurs, le taux d'infestation de $H$. multicinctus a été supérieur à $1000 \mathrm{~N} / \mathrm{dm}^{3}$ de sol, à l'exception du producteur Sa07, avec $225 \mathrm{~N} / \mathrm{dm}^{3}$ de sol. Par rapport aux deux principaux nématodes, les cinq autres nématodes ont eu des taux d'infestation assez faibles allant en moyenne de 1 à $258 \mathrm{~N} / \mathrm{dm}^{3}$ de sol.

\section{Nématodes des racines}

H. multicinctus, $S$. cavenessi et $P$. bachyurus ont été les trois espèces de nématodes rencontrées dans les racines des producteurs du G I, avec des taux d'infestations de 0 à $6 \mathrm{~N} / \mathrm{g}$ de racines (Tableau 5). La plus forte infestation a été due à $S$. cavenessi et a été rencontrée chez le producteur Go01 du groupe avec $6 \mathrm{~N} / \mathrm{g}$ de racines. H. multicinctus a été responsable de deux infestations chez les producteurs Sa14 et Sa15 avec 01 N/g racine. La troisième espèce présente dans les racines a été $P$. brachyurus avec $1 \mathrm{~N} / \mathrm{g}$ de racines chez un seul producteur (Sa21).

Dans les racines du niébé chez les 10 producteurs du G II, deux espèces de nématodes ont été retrouvées (H. multicinctus et $S$. cavenessi), respectivement chez 03 et 04 producteurs (Tableau 6). L'infestation la plus forte par $H$. multicinctus s'est située chez le producteur $S a 10$, avec $8 \mathrm{~N} / \mathrm{g}$ racine, et celle de S. cavenessi se situe chez Go16 et Vi16, avec $02 \mathrm{~N} / \mathrm{g}$ racine. La troisième espèce de nématode observée dans les racines est $P$. brachyurus, qui était présente chez les producteurs Go16 et Vi16 avec 1 N/g racine.

Aucune infestation de nématodes n'a été constatée dans les racines du niébé chez les producteurs du G III (Tableau 7).

L'infestation des racines du niébé par les nématodes au sein du G IV est constatée chez le producteur $\mathrm{Sa} 02$ (Tableau 8). Elle était due à $H$. multicinctus et $S$. cavenessi avec 01 $\mathrm{N} / \mathrm{g}$ de racines. 
Tableau 1: Infestation en nématodes du sol $\left(\mathrm{N} / \mathrm{dm}^{3}\right.$ de sol) sous culture de niébé chez des producteurs appliquant 0 à $20 \mathrm{~kg} / \mathrm{ha}$ de NPK (14-23-14).

\begin{tabular}{|c|c|c|c|c|c|c|c|}
\hline \multirow{2}{*}{$\begin{array}{l}\text { Codes des } \\
\text { producteurs }\end{array}$} & \multicolumn{7}{|c|}{ Espèces de nématodes } \\
\hline & $\begin{array}{c}P . \\
\text { brachyurus }\end{array}$ & $\begin{array}{c}H . \\
\text { multicinctus }\end{array}$ & $\begin{array}{c}S . \\
\text { cavenessi }\end{array}$ & $\begin{array}{c}T . \\
\text { martini }\end{array}$ & $\begin{array}{l}X . \\
s p\end{array}$ & $\begin{array}{c}T . \\
\text { indicus }\end{array}$ & $\begin{array}{c}C . \\
\text { curvatum }\end{array}$ \\
\hline & & & & & & $1967^{\mathrm{a}} \pm 1$ & \\
\hline $\mathrm{Sa} 26$ & $0 \pm 0$ & $3340^{\mathrm{a}} \pm 227$ & $1327^{\mathrm{a}} \pm 90$ & $0 \pm 0$ & $0 \pm 0$ & 34 & $53^{\mathrm{a}} \pm 4$ \\
\hline Sa17 & $3453^{\mathrm{a}} \pm 235$ & $4140^{\mathrm{b}} \pm 282$ & $693^{\mathrm{b}} \pm 47$ & $287^{\mathrm{a}} \pm 19$ & $20^{\mathrm{a}} \pm 1$ & $0 \pm 0$ & $287^{b} \pm 19$ \\
\hline $\mathrm{Sa} 22$ & $0 \pm 0$ & $3408^{\mathrm{a}} \pm 232$ & $416^{\mathrm{b}} \pm 28$ & $128^{\mathrm{b}} \pm 9$ & $0 \pm 0$ & $0 \pm 0$ & $56^{\mathrm{a}} \pm 4$ \\
\hline Sa16 & $13^{\mathrm{b}} \pm 1$ & $907^{\mathrm{c}} \pm 62$ & $266^{\mathrm{c}} \pm 18$ & $373^{c} \pm 25$ & $0 \pm 0$ & $167^{\mathrm{b}} \pm 11$ & $7^{\mathrm{c}} \pm 0$ \\
\hline Sa14 & $7^{\mathrm{b}} \pm 0$ & $700^{c} \pm 48$ & $927^{\mathrm{d}} \pm 63$ & $0 \pm 0$ & $0 \pm 0$ & $0 \pm 0$ & $0 \pm 0$ \\
\hline $\mathrm{Sa} 21$ & $0 \pm 0$ & $140^{\mathrm{d}} \pm 10$ & $1184^{\mathrm{d}} \pm 81$ & $416^{c} \pm 25$ & $0 \pm 0$ & $0 \pm 0$ & $0 \pm 0$ \\
\hline Sa15 & $127^{\mathrm{c}} \pm 9$ & $1820^{\mathrm{i}} \pm 124$ & $907^{\mathrm{d}} \pm 62$ & $153^{\mathrm{b}} \pm 10$ & $120^{\mathrm{b}} \pm 8$ & $0 \pm 0$ & $0 \pm 0$ \\
\hline Go01 & $107^{\mathrm{c}} \pm 7$ & $2933^{\mathrm{a}} \pm 199$ & $1913^{\mathrm{e}} \pm 130$ & $0 \pm 0$ & $0 \pm 0$ & $0 \pm 0$ & $0 \pm 0$ \\
\hline Go27 & $33^{\mathrm{d}} \pm 2$ & $380^{\mathrm{f}} \pm 26$ & $300^{\mathrm{c}} \pm 20$ & $107^{\mathrm{b}} \pm 7$ & $14^{\mathrm{a}} \pm 10$ & $93^{c} \pm 6$ & $0 \pm 0$ \\
\hline Vi05 & $7^{\mathrm{b}} \pm 0$ & $247^{\mathrm{f}} \pm 17$ & $113^{\mathrm{f}} \pm 8$ & $33^{\mathrm{d}} \pm 2$ & $0 \pm 0$ & $33^{\mathrm{d}} \pm 2$ & $0 \pm 0$ \\
\hline Vi19 & $93^{\mathrm{c}} \pm 0$ & $1540^{\mathrm{e}} \pm 105$ & $1567^{\mathrm{a}} \pm 107$ & $140^{\mathrm{b}} \pm 10$ & $13^{\mathrm{a}} \pm 1$ & $7^{\mathrm{e}} \pm 0$ & $0 \pm 0$ \\
\hline Vi18 & $7^{\mathrm{b}} \pm 0$ & $1367^{\mathrm{e}} \pm 93$ & $680^{\mathrm{b}} \pm 46$ & $233^{\mathrm{a}} \pm 16$ & $13^{\mathrm{a}} \pm 1$ & $7^{\mathrm{e}} \pm 0$ & $0 \pm 0$ \\
\hline Go20 & $213^{\mathrm{f}} \pm 15$ & $4980^{\mathrm{g}} \pm 339$ & $147^{\mathrm{f}} \pm 10$ & $0 \pm 0$ & $13^{\mathrm{a}} \pm 1$ & $0 \pm 0$ & $0 \pm 0$ \\
\hline
\end{tabular}

Tableau 2: Infestation en nématodes du sol $\left(\mathrm{N} / \mathrm{dm}^{3} \mathrm{de}\right.$ sol) sous culture de niébé chez des producteurs appliquant 20 à $40 \mathrm{~kg} / \mathrm{ha}$ de NPK (14-23-14).

\begin{tabular}{|c|c|c|c|c|c|c|c|}
\hline \multirow{2}{*}{$\begin{array}{l}\text { Codes des } \\
\text { producteurs }\end{array}$} & \multicolumn{7}{|c|}{ Espèces de nématodes } \\
\hline & $\begin{array}{c}P . \\
\text { brachyurus }\end{array}$ & $\begin{array}{c}H . \\
\text { multicinctus }\end{array}$ & $\begin{array}{c}S . \\
\text { cavenessi }\end{array}$ & $\begin{array}{c}T . \\
\text { martini }\end{array}$ & $\begin{array}{l}X . \\
s p\end{array}$ & $\begin{array}{c}T . \\
\text { indicus }\end{array}$ & $\begin{array}{c}\text { C. } \\
\text { curvatum }\end{array}$ \\
\hline $\mathrm{Sa} 23$ & $0 \pm 0$ & $427^{\mathrm{f}} \pm 28$ & $87^{i} \pm 6$ & $27^{\mathrm{d}} \pm 2$ & $0 \pm 0$ & $0 \pm 0$ & $0 \pm 0$ \\
\hline $\mathrm{Sa} 08$ & $633^{\mathrm{g}} \pm 41$ & $1653^{\mathrm{e}} \pm 107$ & $1240^{\mathrm{a}} \pm 81$ & $127^{\mathrm{b}} \pm 8$ & $320^{c} \pm 21$ & $0 \pm 0$ & $20^{\mathrm{d}} \pm 1$ \\
\hline $\mathrm{Sa} 30$ & $7^{\mathrm{b}} \pm 0$ & $1113^{\mathrm{e}} \pm 72$ & $1513^{\mathrm{a}} \pm 98$ & $20^{\mathrm{d}} \pm 1$ & $0 \pm 0$ & $813^{\mathrm{f}} \pm 53$ & $0 \pm 0$ \\
\hline Sa10 & $180^{\mathrm{f}} \pm 12$ & $7180^{\mathrm{h}} \pm 467$ & $880^{\mathrm{d}} \pm 57$ & $760^{\mathrm{e}} \pm 49$ & $680^{\mathrm{d}} \pm 44$ & $13^{\mathrm{e}} \pm 1$ & $20^{\mathrm{d}} \pm 1$ \\
\hline $\mathrm{Sa} 20$ & $33^{\mathrm{d}} \pm 2$ & $1267^{\mathrm{e}} \pm 82$ & $353^{\mathrm{c}} \pm 23$ & $187^{\mathrm{b}} \pm 12$ & $0 \pm 0$ & $280^{\mathrm{g}} \pm 18$ & $7^{\mathrm{c}} \pm 0$ \\
\hline Go30 & $33^{\mathrm{d}} \pm 2$ & $5373^{\mathrm{g}} \pm 349$ & $727^{\mathrm{b}} \pm 47$ & $0 \pm 0$ & $0 \pm 0$ & $67^{\mathrm{c}} \pm 4$ & $0 \pm 0$ \\
\hline Sa12 & $0 \pm 0$ & $473^{\mathrm{f}} \pm 31$ & $313^{\mathrm{c}} \pm 20$ & $20^{\mathrm{d}} \pm 1$ & $0 \pm 0$ & $0 \pm 0$ & $0 \pm 0$ \\
\hline Go16 & $207^{\mathrm{f}} \pm 13$ & $1827^{\mathrm{e}} \pm 119$ & $2047^{\mathrm{e}} \pm 133$ & $7^{\mathrm{f}} \pm 0$ & $307^{\mathrm{c}} \pm 20$ & $120^{\mathrm{b}} \pm 8$ & $0 \pm 0$ \\
\hline Vi16 & $0 \pm 0$ & $2207^{\mathrm{i}} \pm 143$ & $553^{\mathrm{b}} \pm 36$ & $0 \pm 0$ & $0 \pm 0$ & $40^{\mathrm{d}} \pm 3$ & $0 \pm 0$ \\
\hline Vi13 & $80^{c} \pm 5$ & $2993^{\mathrm{a}} \pm 195$ & $2773^{\mathrm{h}} \pm 180$ & $20^{\mathrm{d}} \pm 1$ & $27^{\mathrm{a}} \pm 2$ & $0 \pm 0$ & $0 \pm 0$ \\
\hline
\end{tabular}
test de Fisher. 
Tableau 3: Infestation en nématodes du sol $\left(\mathrm{N} / \mathrm{dm}^{3} \mathrm{de}\right.$ sol) sous culture de niébé chez des producteurs appliquant 40 à $60 \mathrm{~kg} / \mathrm{ha}$ de NPK (14-23-14).

\begin{tabular}{lccccccc}
\hline & \multicolumn{7}{c}{ Espèces de nématodes } \\
\cline { 2 - 8 } Codes des & $\boldsymbol{P}$. & $\boldsymbol{H}$. & $\boldsymbol{S}$. & $\boldsymbol{T}$. & $\boldsymbol{X}$. & $\boldsymbol{T}$. & $\boldsymbol{C}$. \\
producteurs & brachyurus & multicinctus & cavenessi & martini & $\boldsymbol{s} \boldsymbol{p}$ & indicus & curvatum \\
\hline Sa05 & $7^{\mathrm{b}} \pm 0$ & $3933^{\mathrm{b}} \pm 224$ & $327^{\mathrm{c}} \pm 19$ & $227^{\mathrm{a}} \pm 13$ & $87^{\mathrm{b}} \pm 5$ & $13^{\mathrm{e}} \pm 1$ & $0 \pm 0$ \\
Sa19 & $20^{\mathrm{d}} \pm 1$ & $773^{\mathrm{c}} \pm 44$ & $647^{\mathrm{b}} \pm 37$ & $60^{\mathrm{g}} \pm 3$ & $0 \pm 0$ & $20^{\mathrm{d}} \pm 1$ & $13^{\mathrm{c}} \pm 1$ \\
Go21 & $7^{\mathrm{b}} \pm 0$ & $2993^{\mathrm{a}} \pm 171$ & $840^{\mathrm{d}} \pm 48$ & $7^{\mathrm{f}} \pm 0$ & $0 \pm 0$ & $0 \pm 0$ & $0 \pm 0$
\end{tabular}

Les chiffres portant une même lettre dans la même colonne ne sont pas significativement différents au seuil p<0,05, selon le test de Fisher.

Tableau 4: Infestation en nématodes du sol $\left(\mathrm{N} / \mathrm{dm}^{3}\right.$ de sol) sous culture de niébé chez des producteurs appliquant 60 à $80 \mathrm{~kg} / \mathrm{ha}$ de NPK (14-23-14).

\begin{tabular}{|c|c|c|c|c|c|c|c|}
\hline \multirow[b]{2}{*}{$\begin{array}{l}\text { Codes des } \\
\text { producteurs }\end{array}$} & \multicolumn{7}{|c|}{ Espèces de nématodes } \\
\hline & $\begin{array}{c}P . \\
\text { brachyurus }\end{array}$ & $\begin{array}{c}\mathrm{H} . \\
\text { multicinctu } \\
\mathrm{S}\end{array}$ & $\begin{array}{c}S . \\
\text { cavenessi }\end{array}$ & $\begin{array}{c}T . \\
\text { martini }\end{array}$ & $\begin{array}{l}X . \\
s p\end{array}$ & $\begin{array}{c}T . \\
\text { indicus }\end{array}$ & $\begin{array}{c}C . \\
\text { curvatum }\end{array}$ \\
\hline & & & & $551^{\mathrm{h}} \pm 4$ & & & \\
\hline Sa07 & $0 \pm 0$ & $225^{\mathrm{f}} \pm 16$ & $\begin{array}{l}1256^{\mathrm{a}} \pm 90 \\
2793^{\mathrm{h}} \pm 20\end{array}$ & $\begin{array}{c}0 \\
533^{\mathrm{h}} \pm 3\end{array}$ & $0 \pm 0$ & $0 \pm 0$ & $0 \pm 0$ \\
\hline Sa02 & $507^{\mathrm{g}} \pm 36$ & $7000^{\mathrm{h}} \pm 504$ & $\begin{array}{c}1 \\
3033^{\mathrm{h}} \pm 21\end{array}$ & $\begin{array}{c}8 \\
327^{c} \pm 2\end{array}$ & $13^{\mathrm{a}} \pm 1$ & $0 \pm 0$ & $0 \pm 0$ \\
\hline Sa09 & $27^{\mathrm{d}} \pm 2$ & $4193^{b} \pm 302$ & 8 & 4 & $80^{\mathrm{b}} \pm 6$ & $0 \pm 6$ & $0 \pm 0$ \\
\hline Sa03 & $0 \pm 0$ & $2140^{\mathrm{i}} \pm 154$ & $1200^{\mathrm{a}} \pm 86$ & $13^{\mathrm{f}} \pm 1$ & $0 \pm 0$ & $60^{\mathrm{c}} \pm 4$ & $7^{\mathrm{c}} \pm 0$ \\
\hline Go24 & $320^{\mathrm{h}} \pm 23$ & $5293^{\mathrm{g}} \pm 381$ & $707^{\mathrm{b}} \pm 51$ & $33^{\mathrm{d}} \pm 2$ & $27^{\mathrm{a}} \pm 2$ & $253^{\mathrm{g}} \pm 18$ & $0 \pm 0$ \\
\hline Go29 & $93^{c} \pm 7$ & $3040^{\mathrm{a}} \pm 219$ & $293^{c} \pm 21$ & $93^{\mathrm{g}} \pm 7$ & $27^{\mathrm{a}} \pm 2$ & $20^{\mathrm{d}} \pm 1$ & $0 \pm 0$ \\
\hline
\end{tabular}

Les chiffres portant une même lettre dans la même colonne ne sont pas significativement différents au seuil $p<0,05$, selon le test de Fisher. 
Tableau 5: Infestation en nématodes des racines (N/g de racines) sous culture de niébé chez des producteurs appliquant 0 à $20 \mathrm{~kg} / \mathrm{ha}$ de NPK (14-23-14).

\begin{tabular}{lccc}
\hline \multirow{2}{*}{ Codes des producteurs } & \multicolumn{3}{c}{ Espèces de nématodes } \\
\cline { 2 - 4 } & $\boldsymbol{P}$. brachyurus & H. multicinctus & S. cavanessi \\
\hline Sa26 & $0 \pm 0$ & $0 \pm 0$ & $0 \pm 0$ \\
Sa17 & $0 \pm 0$ & $0 \pm 0$ & $0 \pm 0$ \\
Sa22 & $0 \pm 0$ & $0 \pm 0$ & $0 \pm 0$ \\
Sa16 & $0 \pm 0$ & $0 \pm 0$ & $0 \pm 0$ \\
Sa14 & $0 \pm 0$ & $1^{\mathrm{a}} \pm 0$ & $0 \pm 0$ \\
Sa21 & $1^{\mathrm{a}} \pm 0$ & $0 \pm 0$ & $0 \pm 0$ \\
Sa15 & $0 \pm 0$ & $1^{\mathrm{a}} \pm 0$ & $2^{\mathrm{a}} \pm 0$ \\
Go01 & $0 \pm 0$ & $0 \pm 0$ & $6^{\mathrm{b}} \pm 0$ \\
Go27 & $0 \pm 0$ & $0 \pm 0$ & $0 \pm 0$ \\
Vi05 & $0 \pm 0$ & $0 \pm 0$ & $0 \pm 0$ \\
Vi19 & $0 \pm 0$ & $0 \pm 0$ & $0 \pm 0$ \\
Vi18 & $0 \pm 0$ & $0 \pm 0$ & $0 \pm 0$ \\
Go20 & $0 \pm 0$ & $0 \pm 0$ & $0 \pm 0$ \\
\hline
\end{tabular}

Les chiffres portant une même lettre dans la même colonne ne sont pas significativement différents au seuil $p<0,05$, selon le test de Fisher.

Tableau 6: Infestation en nématodes des racines (N/g de racines) sous culture de niébé chez des producteurs appliquant 20 à $40 \mathrm{~kg} / \mathrm{ha}$ de NPK (14-23-14).

\begin{tabular}{lccc}
\hline \multirow{2}{*}{ Codes des producteurs } & \multicolumn{3}{c}{ Espèces de nématodes } \\
\cline { 2 - 4 } & P. brachyurus & H. multicinctus & S. cavanessi \\
\hline Sa23 & $0 \pm 0$ & $0 \pm 0$ & $0 \pm 0$ \\
Sa08 & $0 \pm 0$ & $0 \pm 0$ & $0 \pm 0$ \\
Sa30 & $0 \pm 0$ & $0 \pm 0$ & $0 \pm 0$ \\
Sa10 & $0 \pm 0$ & $8^{\mathrm{b}} \pm 1$ & $1^{\mathrm{a}} \pm 0$ \\
Sa20 & $0 \pm 0$ & $0 \pm 0$ & $0 \pm 0$ \\
Go30 & $0 \pm 0$ & $1^{\mathrm{a}} \pm 0$ & $0 \pm 0$ \\
Sa12 & $0 \pm 0$ & $0 \pm 0$ & $0 \pm 0$ \\
Go16 & $1^{\mathrm{a}} \pm 0$ & $2^{\mathrm{a}} \pm 0$ \\
Vi16 & $0 \pm 0$ & $2^{\mathrm{a}} \pm 0$ \\
Vi13 & $1^{\mathrm{a}} \pm 0$ & $0 \pm 0$ & $1^{\mathrm{a}} \pm 0$ \\
\hline Les chiffres portant une même lettre dans la même colonne ne sont pas significativement différents au seuil $\mathrm{p}<0,05$, selon le \\
test de Fisher.
\end{tabular}

Tableau 7: Infestation en nématodes des racines (N/g de racines) sous culture de niébé chez des producteurs appliquant 40 à $60 \mathrm{~kg} / \mathrm{ha}$ de NPK (14-23-14). 


\begin{tabular}{lccc}
\hline Codes des producteurs & \multicolumn{3}{c}{ Espèces de nématodes } \\
\cline { 2 - 4 } & $\boldsymbol{P}$. brachyurus & H. multicinctus & S. cavanessi \\
\hline Sa05 & $0 \pm 0$ & $0 \pm 0$ & $0 \pm 0$ \\
Sa19 & $0 \pm 0$ & $0 \pm 0$ & $0 \pm 0$ \\
Go21 & $0 \pm 0$ & $0 \pm 0$ & $0 \pm 0$ \\
\hline
\end{tabular}

Les chiffres portant une même lettre dans la même colonne ne sont pas significativement différents au seuil $\mathrm{p}<0,05$, selon le test de Fisher.

Tableau 8: Infestation en nématodes des racines (N/g de racines) sous culture de niébé chez des producteurs appliquant 60 à $80 \mathrm{~kg} / \mathrm{ha}$ de NPK (14-23-14).

\begin{tabular}{lccc}
\hline \multirow{2}{*}{ Codes des producteurs } & \multicolumn{3}{c}{ Espèces de nématodes } \\
\cline { 2 - 4 } & $\boldsymbol{P .}$ brachyurus & H. multicinctus & S. cavanessi \\
\hline Sa07 & $0 \pm 0$ & $0 \pm 0$ & $0 \pm 0$ \\
Sa02 & $0 \pm 0$ & $1^{\mathrm{a}} \pm 0$ & $1^{\mathrm{a}} \pm 0$ \\
$\mathrm{Sa} 09$ & $0 \pm 0$ & $0 \pm 0$ & $0 \pm 0$ \\
$\mathrm{Sa} 03$ & $0 \pm 0$ & $0 \pm 0$ & $0 \pm 0$ \\
Go24 & $0 \pm 0$ & $0 \pm 0$ & $0 \pm 0$ \\
Go29 & $0 \pm 0$ & $0 \pm 0$ & $0 \pm 0$ \\
\hline
\end{tabular}

Les chiffres portant une même lettre dans la même colonne ne sont pas significativement différents au seuil $\mathrm{p}<0,05$, selon le test de Fisher.

\section{DISCUSSION}

Nématodes du sol

Dans les exploitations paysannes sous culture de niébé que nous avons étudiées, nous avons recensé sept espèces de nématodes dont cinq appartiennent à des genres dominants qui sont les plus caractéristiques de la région sahélienne, à savoir: les genres Scutellonema ( $S$. cavenessi), Pratylenchus ( $P$. brachyurus), Helicotylenchus (H. multicinctus), Tylenchorhynchus (T. martini) et Telotylenchus ( $T$. indicus). Ils font partie des nématodes d'importance économique (Taylor, 1976 ; Clafin, 1984, Sikora et al., 2005b). Baujard (1986) note qu'ils sont capables de se multiplier sur la plupart des plantes cultivées et les plantes sauvages pendant l'inter-culture. Nous avons constaté que la fertilisation n'a pas eu d'effet sur les nématodes du sol. Si nous considérons les deux principaux nématodes responsables des infestations que sont $H$. multicinctus, $S$. cavenessi, les niveaux des infestations sont restés souvent sensiblement les mêmes entre parcelles de niveaux de fertilisation différents au sein des 04 groupes de fertilisation. La fertilisation en NPK et urée n'a pas affecté de façon sensible les populations de nématodes du sol. Ces résultats corroborent les travaux des auteurs comme Gnonhouri et Diomandé (1989) et Sundararaju et Kumar, (2003) qui ont montré qu'un apport d'engrais azotés et phosphorés seuls n'a pas eu d'effet sur les populations des nématodes du sol.

\section{Nématodes des racines}

Nos résultats montrent une très faible infestation des racines du niébé. Les infestations des racines constatées sont dues à trois nématodes, $P$. brachyurus, $H$. 
multicinctus et $S$. cavenessi. Les nématodes du sol qui ont des taux d'infestation assez élevés sont quasiment absents dans les racines des cultures. Ceci confirme l'hypothèse selon laquelle la fertilisation azotée vise, non pas à lutter directement contre les nématodes phytoparasites, mais à donner une plus grande vigueur à la plante pour se défendre ou encore lui permettre de modifier l'équilibre biopédologique au détriment du parasite comme le font la plupart des substances nématicides (Sarah et Hugon, 1991; Dmowska et Ilieva, 1995). L'azote apporté à la plante se retrouve dans la zone du chevelu racinaire, améliore la possibilité d'absorption des racines et empêche les nématodes de pénétrer dans la plante (Mateille, 1994 ; Sikora et al., 2005b). Cela a eu pour conséquence une très faible infestation des racines du niébé par les nématodes. En plus de la fertilisation apportée de manière exogène, certaines propriétés de la plante du niébé pourraient être également à l'origine de cette faible infestation de ses racines par les nématodes. Le niébé, comme les autres légumineuses, a des besoins en sources externes d'azote peu élevés, car ses racines sont munies de nodosités peuplées de bactéries (Rhizobiums) qui contribuent à la fixation de l'azote atmosphérique. Le Rhizobium est une bactérie qui infecte les racines des légumineuses et donne naissance à des excroissances de type tumoral appelées nodosités. Le centre de chaque nodosité mature contient des milliards de bactéries qui fixent l'azote. La légumineuse hôte fournit l'énergie nécessaire à cette fixation en capturant l'énergie du soleil par le phénomène de la photosynthèse. A cause de sa capacité de fixation d'azote par voie symbiotique, on estime en effet à $30 \mathrm{~kg}$ de NPK/ha les besoins en fertilisation du niébé (Dugje et al., 2009 ; Osei et al., 2011). Ces résultats peuvent s'expliquer aussi par l'absence de nématodes cécidogènes (Meloidogyne spp.) dans le sol de ces exploitations. Les nématodes cécidogènes sont en effet considérés comme principaux ravageurs du niébé à l'échelle mondiale (Sikora et al., 2005; James et al., 2010 ; Castagnone-Sereno et Djian-Caporalino, 2011).

\section{Conclusion}

La nématofaune au niveau des 32 exploitations de niébé étudiées est composée de 07 espèces dont 02 espèces principales, $H$. multicinctus et Scutellonema cavenessi. Le nématode à galles, Meloidogyne spp. qui est le principal nématode inféodé au niébé n'a pas été rencontré au cours de cette étude. L'infestation des racines par les nématodes a été constatée dans 04 exploitations et est due à trois espèces de nématodes, $H$. multicinctus, $S$. cavenessi et $P$. brachyurus. Ces infestations sont constatées dans neuf cas sur dix dans les exploitations avec une fertilisation minérale de 0 à $40 \mathrm{~kg}$ de NPK (GI, GII). Un seul cas d'infestation des racines est constaté avec la fumure minérale aux doses supérieures à 40 $\mathrm{kg} / \mathrm{ha}$ (GIII, GIV). L'utilisation de la fumure minérale a fait baisser la pression des nématodes sur les cultures.

\section{REMERCIEMENTS}

Nous remercions les techniciens de recherche, Sanou Martin à la Station de Recherches Agricoles de Saria et Kièmdé Salam au Laboratoire de Nématologie du Centre Régional de Recherches Environnementales et Agricoles de Bobo Dioulasso pour leur appui lors des travaux de terrain et d'extraction des nématodes. Nous remercions également Diallo Boukary Ousmane, Maître de Recherches à l'Institut de l'Environnement et de Recherches Agricoles pour son appui dans le traitement statistiques des données.

\section{REFERENCES}

Bachelier G. 1978. La Faune du Sol, son Ecologie et son Action. OSRTOM: Paris, France. 
Bado BV. 2002. Rôle des légumineuses sur la fertilité des sols ferrugineux tropicaux des zones guinéenne et soudanienne du Burkina Faso. Thèse de Doctorat, Université Laval, département des sols et de génie agroalimentaire, Québec, Canada, 197 p.

Baujard P. 1986. Écologie des nématodes dans le bassin arachidier du Sénégal. Revue Nématol., 9: 288-302.

Bélair G. 2005. Les nématodes, ces anguillules qui font suer les plantes par la racine. Phytoprotection, 86(1): 65-69.

Bois JF, Cadet P, Plenchette CH, Duponnois R. 2000. Impact des nématodes phytoparasites de la zone Soudano Sahélienne du Sénégal sur la croissance du mil en conditions contrôlées. Étude et Gestion des Sols, 7(4): 271-278.

Cadet P. 1998. Gestion écologique des nématodes phytoparasites tropicaux. Cahier d'Agriculture, 7: 187-194.

Castagnone-Sereno P, Djian-Caporalino C. 2011. Lutte contre les nématodes à galles en cultures maraîchères : des recherches pour promouvoir la durabilité des résistances variétales. Innovations Agronomiques, 15: 55-64.

Celetti MJ. 2006. Échantillonnage du Sol et des Racines Visant le Dénombrement des Nématodes Phytoparasites. Fiche Technique, Edition la Reine pour l'Ontario : Canada.

Clafin LE. 1984. Plant-parasitic nematodes affecting sorghum. In Sorghum Root and Stalk Rots, a Critical Review: Proceedings of the Consultative Group Discussion on Research Needs and Strategies for Control of Sorghum Root and Stalk Rot Diseases. 27 Nov - 2 Décembre 1983 Bellagio, Italy, ICRISAT, 53-58.

Dmowka E, Ilieva K. 1995. The effect of prolonged diverse mineral fertilization on nematodes inhabiting the rhizosphere of spring barley. European Journal of Soil Biology, 31(4): 189-198.

Dugje IY, Omoigui LO, Ekeleme F, Kamara AY, Ajeigbe H. 2009. Production $d u$ Niébé en Afrique de l'Ouest: Guide du Paysan. Institut International d'Agriculture Tropicale (IITA): Ibadan, Nigeria.

FAO. 2010. FAOSTAT/Agriculture., http://faostat.fao.org/site/567/DesktopDef ault.aspx?PageID=567\#ancor (26 mai 2013).

Gnonhouri P, Diomandé M. 1989. Action de la Fertilisation et des Nématicides non Fumigants sur les Populations de Nématodes et le Rendement Paddy à Yabra. ORSTOM-AISA : Adiopodoumé, Abidjan, Côte d'Ivoire.

Hien E. 2004. Dynamique du carbone dans un Acrisol ferrique du Centre Ouest Burkina: Influence des pratiques culturales sur le stock et la qualité de la matière organique. Thèse de Doctorat, Ecole Nationale Supérieure Agronomique de Montpellier, France, 140 p.

INSD. 2009b. Monographie des Régions du Burkina Faso. Ministère de l'Économie et des Finances (MEF), Recensement Général de la population et de l'Habitat de 2006 (RGPH-2006): Ouagadougou, Burkina Faso.

James B, Atcha-Ahowé C, Godonou I, Baimey H, Goergen G, Sikirou R, Toko M. 2010. Gestion Intégrée des Nuisibles en Production Maraîchère : Guide pour les Agents de Vulgarisation en Afrique de l'Ouest. Institut international d'agriculture tropicale (IITA): Ibadan, Nigeria.

Kabore M, Yameogo O, Compaore F, Fyato M, Ouédraogo A, Ouédraogo M. 2010. Analyse de la production agricole au Burkina Faso à partir de la base de données CountrySTAT Burkina (1984 à 2009). In Le bulletin mensuel d'information sur CountrySTAT Burkina 
Faso $n^{\circ} 3$. Direction Générale de la Promotion de l'Économie Rurale (DGPER) : Ouagadougou, Burkina Faso ; 1-3.

Lompo F. 2009. Effets induits des modes de gestion de la fertilité sur les états du phosphore et la solubilité des phosphates naturels dans deux sols du Burkina Faso. Thèse de Doctorat d'État ès-Sciences Naturelles, Université de Cocody, Côte d'Ivoire, $178 \mathrm{p}$.

Luc M. 1960. Les nématodes associés aux plantes de l'Ouest Africain, Liste préliminaire. L'Agronomie Tropicale, 15(4): 434-449.

Mallamaire A. 1965. Deux nématodes nuisibles aux plantes cultivées au Sénégal. In Collection de référence, Sér. Protection des Cultures Tropicales. ORSTOM : Paris, France ; 689 - 694.

Mateille T. 1994. Biologie de la relation plantes-nématodes: perturbations physiologiques et mécanismes de défense des plantes. Nematologica, 40: 276-311.

McDonald HA, Nicol MJ. 2005. Nematodes Parasites of Cereals. In Plant Parasitic Nematodes in Subtropical and Tropical Agriculture, Luc M, Sikora RA, Bridge J (eds). CABI Publishing: Wageningen, The Netherlands; 131-192.

Merny G, Luc M. 1969. Les techniques d'échantillonnages des peuplements de nématodes ans le sol. In Problèmes d'Écologie: l'Echantillonnage des Peuplements d'Animaux des Milieux Terrestres, Lamotte M, Bourlière F (éds). Masson \& Cie : Paris ; 236-272.

Osei K, Moss R, Nafeo A, Addico R, Agyemang A, Danso Y, Asante JS. 2011. Management of plant parasitic nematodes with antagonistic plants in the forestsavanna transitional zone of Ghana. Journal of Applied Biosciences 37: 24912495.
Ouédraogo S. 2005. Intensification de l'agriculture dans le plateau central du Burkina Faso : Une Analyse des possibilités à partir des nouvelles Technologies. Thèse de PhD, Rijksuniversiteit Groningen, $336 \mathrm{p}$.

Prot JC. 1985. Importance des nématodes phytoparasites en zones Sahélienne. In Institut du Sahel. Lettre d'Information n¹3, Bamako, Mali, 3-6.

Reversat G. 1988. Implication des nématodes phytoparasites dans le concept de la fatigue des sols. In Rapport du Séminaire International de Pointe Noire sur le thème : Facteurs et Conditions de la Fertilité du Milieu Tropical Humide. UNESCOPNUD-AUPELF. Paris, France, 123-133.

Sarah JL, Hugon R. 1991. Dynamique des populations Pratylenchus brachyurus en plantation d'ananas en Côte d'Ivoire. Fruits, 46(3): 241-250.

Seinhorst JW. 1998. The common relation between population density and plant weight in pot and microplot experiments with various nematode plant combinations Fundam. Appl. Nematol., 21(5): 459-468.

Sedogo PM. 1993. Évolution des sols ferrugineux lessivés sous culture: incidence des modes de gestion sur la fertilité. Thèse de Doctorat ès Sciences, Université Nationale de Côte d'Ivoire, Abidjan, République de Côte d'Ivoire, $330 \mathrm{p}$.

Sikora RA, Greco N, Silva VFJ. 2005a. Management Practices:an Overview of Integrated Nematode Management Technologies. In Plant Parasitic Nematodes in Subtropical and Tropical Agriculture. Luc M, Sikora RA, Bridge J (eds). CABI Publishing: Wageningen, The Netherlands; 259-318.

Sikora RA, Bridge J, Starr LJ. 2005 b. Nematode Parasites of Food Legumes. In Plant Parasitic Nematodes in Subtropical and Tropical Agriculture. Luc M, Sikora 
RA, Bridge J (eds). CABI Publishing: Wageningen, The Netherlands; 793-826.

Sundararaju P, Kumar V. 2003. Gestion de Pratylenchus coffeae au moyen d'amendements organiques et minéraux. InfoMusa, 12(1): 35-39.

Talwana HL, Butseye MM, Tusiime G. 2008. Occurrence of plant parasitic nematodes and factors that enhance population buildup in cereal-based cropping systems in Uganda. African Crop Science Journal, 16(2): 119-131.

Taylor PD. 1976. Plant nematology problems in tropical africa. Plant Nematology, 45(4): 269-284.
Weber A, Oerke EC, Dehne HW, Schonbeck F. 1994. Crop Production and Crop Protection: Estimated Losses in Major Food and Cash Crops. Elsevier Science: USA.

Yeates GW, Bird AF. 1994. Some observations on the influence of agricultural practices on the nematode fauna of some South Australian soils. Fundam. Appl. Nemalol., 17(2) : 133-145. 